\title{
Negro-white differences in attribution of responsibility as a function of age
}

MARVIN E. SHAW, University of Florida, Gainesville, Fla. 32601, and FRANK W. SCHNEIDER, University of Windsor, Windsor, Ontario, Canada

An attribution-of-responsibility (AR) questionnaire was administered to matched samples of Negro and white children representing four age levels. Ethnic differences were observed in the two youngest groups, whereas there were no ethnic differences among the older children. In the younger age groups, Negroes showed a generally less differentiated pattern of $A R$ than the whites. The results were interpreted as supporting the hypothesis that a deprived cultural background retards the rate of learning norms concerning responsibility attribution.

Heider (1958) considered the variables which determine attribution of responsibility (AR). Expanding upon the work of Piaget (1932), Heider outlined five levels in the development of "sophisticated" attribution. At the lowest level of sophistication (Level 1: association), the attributor holds the other person responsible for any outcome with which he is in any way associated. At the next level (Level 2: commission), the attributor holds the other person responsible for any outcome that he produces by his actions. At Level 3 (foreseeability), the other person is held responsible for any foreseeable outcome that he produces. At Level 4 (intentionality), the other person is held responsible for any outcome that he produces intentionally; and at Level 5 (justification), he is held less responsible than at Level 4 if the intended outcome was produced by justified actions (i.e., he acted under coercion or provocation). Thus, in attributing responsibility, unsophisticated persons (e.g., young children) are likely to consider only association, whereas more sophisticated individuals (e.g., adults) take into account more variables.

A study by Shaw \& Sulzer (1964) provided support for Heider's analysis. They found that, in contrast to adults, children were generally less differentiated in their use of the variables outlined by Heider, attributing more at Levels 1 (association) and 2 (commission) and less at Levels 4 (intentionality) and 5 (justification). This study and subsequent studies (e.g., Garcia-Esteve \& Shaw, 1968) also have demonstrated that $A R$ is influenced by the quality of the outcome (i.e., whether the effect of the behavior in question is good or bad) and by the intensity of the outcome (i.e., how good or how bad).

Theoretically, the degree to which an individual considers these structural variables (the levels of causality and the quality and intensity of outcome) in attributing responsibility depends upon the degree to which he has learned the norms of his culture. It follows that the culture in which the attributor is reared should be related to AR. Experimental evidence supports this expectation (Garcia-Esteve \& Shaw, 1968; Shaw, Briscoe, \&

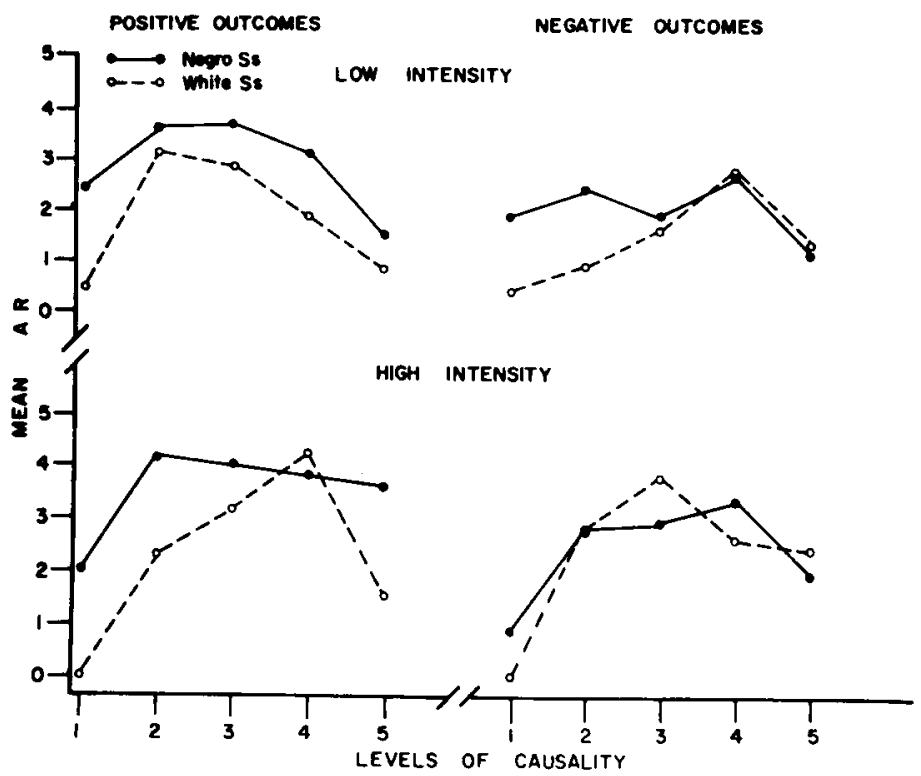

Garcia-Esteve, 1968). One aspect of this analysis, however, has not been explored-the effects of a deprived cultural environment upon the rate of learning the norms concerning AR. In one study, ${ }^{2}$ in southern United States, it was found that second-grade Negroes showed a less sophisticated (less differentiated) AR pattern than second-grade whites; however, this difference did not appear among 6th and 12th graders. Sulzer and Shaw interpreted their findings as due to the relative cultural deprivation experienced by the southern Negro, wherein he has inadequate opportunities to learn the approved mode of responding.

Unfortunately, Sulzer and Shaw failed to control for possible ethnic differences in socioeconomic level and intellectual ability. While the evidence for a significant relationship between these variables and AR is not strong (Shaw \& Schneider, 1967, in press), it would be desirable to control for them. The purpose of the present study was to examine Negro-white differences in AR as a function of age. It was expected that Negro children would display slower development than white children in sophisticated AR. Consequently, ethnic differences in $A R$ were anticipated among younger children but not among older children.

\section{SUBJECTS}

The Ss were drawn from two public schools in rural Florida. One was an all-Negro school, and the other was predominantly white. Four samples were obtained from each school, representing the following age groups: $7-8$ years $(N=12$ per school), 9-10 years $(\mathrm{N}=15$ per school), 11-12 years $(\mathrm{N}=15$ per school), and 17.19 years $(\mathrm{N}=15$ per school). Therefore, within each age group, half of the Ss were white, and half were Negro. The Negro and white samples were matched as closely as possible on socioeconomic status, religion, age, sex, and intelligence. Matching was satisfactory (i.e., no significant differences) on all measures except intelligence. It was not possible to obtain matched Ss on this variable because of the wide differences in measured intelligence between the white Ss (mean $=100.3$ ) and Negro Ss (mean $=89.7)$.

\section{MATERIALS}

The materials were the same as those used in some earlier reports (e.g., Shaw \& Schneider, in press) and similar to the "abstract structures" used by Sulzer (1964).

Measures of AR were obtained by means of a questionnaire which consisted of 20 items designed to represent the five levels of "sophistication" in combination with

Fig. 1. Ethnic differences in attribution of responsibility as a function of structural variables for 7- to 8-year-olds. 


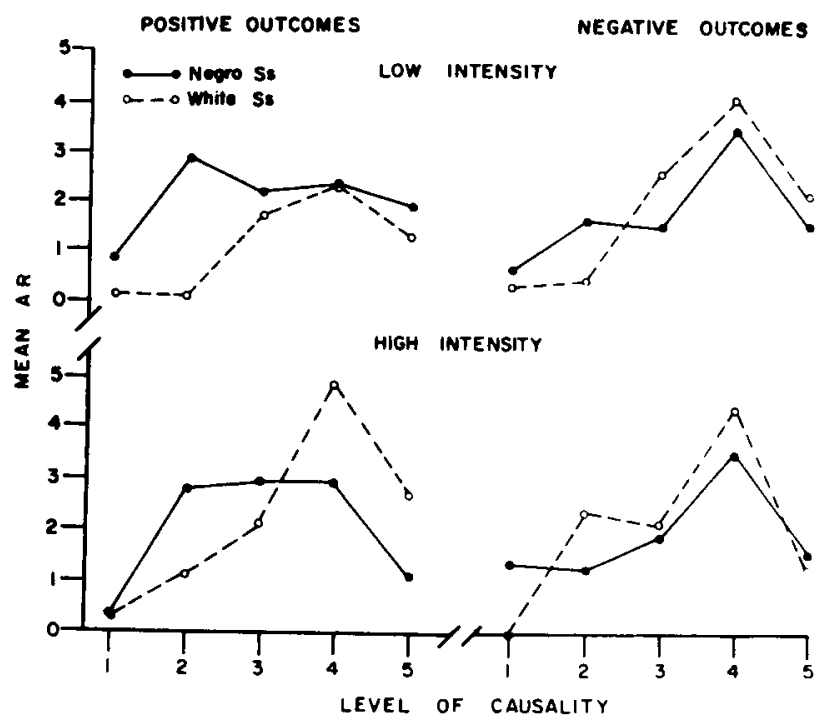

positive or negative outcomes of either high or low intensity. The following is a sample item: "Paul caused something to happen that was very good. He intended to cause it. Is Paul responsible for the very good thing that he caused?" (Level 4: intentionality, positive outcome, high intensity.) A response sheet was provided for each $S$ which required that he circle either YES or NO to indicate whether the agent was responsible for the outcome depicted in each item. If he circled YES, he was required to mark one of five boxes, arranged vertically in descending order of size, to indicate how responsible the agent was for what happened.

\section{PROCEDURE}

Data was collected in the classroom during regular school hours. Each item was read twice before the $S s$ were asked to respond. Data relevant to socioeconomic status, age, sex, intelligence, and religion were obtained from school records. Socioeconomic status was based on Edward's occupational grouping, as described by Miller (1964). Intelligence scores were based on either the Kuhlmann-Finch, the Otis, or the California Test of Mental Maturity.

\section{RESULTS}

AR scores were computed by assigning a value of 0 for each NO response and a value from 1 to 5 for each YES response, with the higher score indicating greater attributed responsibility. Since matching obtained only within age groups, data for each age group were analyzed separately by means of a 2 by 5 by 2 by 2 (ethnic by levels by quality by intensity) analysis of variance.

\section{Ethnic Differences}

In the 7- to 8-year group, an ethnic main effect $(F=5.55, \quad \mathrm{df}=1 / 22, \quad \mathrm{p}<.05)$ indicated Negroes attributed significantly more than whites under all conditions. In the 9- to 10-year group, ethnic differences were revealed by the following interactions: Ethnic by Levels $(\mathrm{F}=3.75, \mathrm{df}=4 / 112$, $\mathrm{p}<.01)$, Ethnic by Levels by Intensity $(F=2.83, \mathrm{df}=4 / 112, \mathrm{p}<.05)$, and Ethnic by Levels by Quality by Intensity $(F=4.14$, $\mathrm{df}=4 / 112, \mathrm{p}<.001)$. No ethnic differences were found in the two older groups.

It may be observed in Fig. 1 that the Negro 7- to 8-year-olds displayed greater AR than did the white 7- to 8-year-olds. The pattern is particularly pronounced for low-intensity, positive outcomes. In the three other conditions, the general trend of higher AR scores for Negroes is clearly evident, although there are a few exceptions, e.g., at Level 4 for high-intensity, positive outcomes, and at Levels 3 and 5 for high-intensity, negative outcomes. In spite of these reversals in ethnic differences, only the overall effect achieved an acceptable level of significance.

Figure 2 reveals that, for the 9- to 10year-old group, ethnic differences varied greatly with levels of causality and appear greater with positive than with negative outcomes. In general, the 9- to 10-year old Negroes displayed a less differentiated AR pattern than the 9- to 10-year-old whites. Notice in Fig. 1 that among the youngest children the trend of less differentiation for Negro Ss also occurred, although it was not as distinctive as in the 9- to 10-year group.

Ethnic differences did not occur in the 10- to 11- and 17- to 19-year groups. There was a marked similarity between the AR curves (not shown) of the older Negroes and whites. Also, the AR patterns of the older Ss reflected greater differentiation than those shown by the two younger groups. Effects of Levels and Outcome Variables

Since the effects of the structural variables (levels of causality, outcome
Fig. 2. Ethnic differences in attribution of responsibility as a function of structural variables for 9- to 10-year-olds.

quality, and outcome intensity) were essentially the same as those found in other studies (Shaw \& Sulzer, 1964; Sulzer, 1964), and since the primary objective of this study concerned ethnic differences in AR, significant sources of variance not involving the ethnic factor will not be reported in detail. $^{3}$ In general, $A R$ increased from Level 1 to Level 4, followed by a decrease at Level 5 . The magnitude of the effect varied with the age and ethnicity of the Ss and with outcome quality and intensity. The typical AR pattern was more pronounced in older $S s$, and the differentiation among levels developed faster among whites than among Negroes. AR generally was greater with negative than with positive outcomes and with high-intensity outcomes than with low-intensity outcomes.

\section{DISCUSSION}

The results of this study generally support the hypothesis that a deprived cultural background retards the rate of learning norms concerning responsibility attribution. Ethnic differences were observed among younger Ss, but these differences were not found among older Ss. It appears that the Negro and white Ss learn to use the same standards (variables) in attributing responsibility, but that the whites learn at a faster rate, i.e., become more differentiating at an early age.

Since it was impossible to match Negro and white $S s$ with respect to measured intelligence, it might be contended that the difference in rate of developing "sophistication" in AR is due to this factor. There are several arguments against this interpretation. First, attempts to demonstrate a relationship between intelligence and AR have generally failed (Shaw \& Schneider, in press). Second, the matching in the present study was much closer in the younger age group, where ethnic differences in AR were found, than in the older age groups, where no differences were found. For example, in the 7- to 8-year group, the mean IQ for Negroes was 104.1, and for whites it was 104.2 ; in the 17- to 19-year group, the mean IQs were 72.5 and 99.9 for Negroes and whites, respectively. For these reasons, ethnic differences in $\mathrm{AR}$ do not appear to be attributable to differences in intelligence. The cultural deprivation hypothesis appears much more tenable.

\section{REFERENCES}

GARCIA-ESTEVE, J., \& SHAW, M. E. Rural and urban pattems of responsibility attribution in Puerto Rico. Journal of Social Psychology, $1968,74,143-149$. 
HEIDER, F. The psychology of interpersonal relations. New York: WiFey, 1958.

MILLER, D. C. Handbook of research design and social measurement. New York: David McKay, 1964.

PIAGET, J. The moral judgment of the child. New York: Harcourt-Brace, 1932.

SHAW, M. E., BRISCOE, M. E., \& GARCIA-ESTEVE, J. A cross-cultural study of attribution of responsibility. International Journal of Psychology, 1968, 3, 51-60.

SHAW, M. E., \& SCHNEIDER, F. W. Social class differences in attribution of responsibility and sanctioning behavior. Research Report No. 5 NSF Grant GS-647, University of Florida, 1967

SHAW, M. E., \& SCHNEIDER, F. W. Intellectua competence as a variable in attribution of responsibility and assignment of sanctions. Journal of Social Psychology, in press.
SHAW, M. E., \& SULZER, J. L. An empirical test of Heider's levels in attribution of responsibility. Journal of Abnormal \& Social Psychology, 1964, 69, 39-46.

SULZER, J. L. Attribution of responsibility as function of the structure, quality, and intensity of the event. Unpublished doctoral dissertation University of Florida, 1964.

\section{NOTES}

1. This research was supported by NSF Grant GS-647. The statistical analyses were done by the University of Florida Computing Center.

2. Sulzer, J. L., \& Shaw, M. E. A comparison of pattems of responsibility attribution in Negroes and whites. Unpublished research, University of Florida, 1964.

3. Readers interested in obtaining a copy of the complete statistical analy ses may write to the first author.

\section{The effects of an irrelevant stimulus variable on transposition}

\author{
NANCY WRAY DAHLEM, Saint Louis \\ University, St. Louis, Mo. 63103
}

On an intermediate size problem, either white cubes were used on both training and test trials, or irrelevant color cues were introduced at the outset of test trials. Analysis of the first test trial data indicated no effect of color on the frequency of transposition responding. Analysis of total test data (18 trials) indicated that color, as a within-trials variable, interfered with transposition.

It has been suggested that the degree of difference among test stimuli and "noticeability" of the change between training and test stimuli are determinants of transposition (Reese, 1968; Zeiler, 1967). Yet, studies concerned with differences within stimulus sets have manipulated only cues on the stimulus dimension relevant to problem solution. Also, only four studies known to the writer have attempted to influence the probability of noticing the change from training to test stimuli by manipulating cues on an irrelevant stimulus dimension. Of these latter studies, one is difficult to interpret because type of cue manipulation used was confounded with the distance of the test (Shirai, 1954). A second offers little general information because retardates were on a partial reinforcement schedule during the training task (Thompson, 1965). Of the two remaining studies, one offers clear support for the idea that failure to notice a training-test difference is crucial for transposition (Stevenson, Langford, \& Reese, 1955) whereas the other offers only questionable support for the idea (Hansen \& Cole, 1968).

The present study investigated the frequency of transposition responses on an intermediate size problem when irrelevant color cues were introduced as one-step (near test) and three-step (far test) trials began. Colors were introduced to test the ideas that degree of difference among test stimuli and "noticeability" of the change from training to test stimuli are factors important to transposition.

\section{METHOD}

The Ss were 150 kindergarteners. ${ }^{2}$ They were randomly assigned to the six conditions of the experiment with the restriction that each condition include an equal number of Ss.

Materials were a 12-in.-high screen which shielded E's hand movements from S's view, a felt-covered stimulus presentation tray, and a series of wooden cubes. The white, red, blue, and yellow cubes measured 1 to $2 \frac{1}{4}$ in. on a side, with cubes adjacent in the series differing from one another by $1 / 4$ in. on a side.

Triads of cubes were presented simultaneously on the stimulus tray via a trapdoor under the screen which separated the $E$ from the $S$. Midpoints of the cubes were aligned with a 2-in. inter-cube separation. Spatial position of the cubes was randomized over trials so that each size cube occupied the left, middle, and right spatial positions twice during each block of six trials. Responses to the middle-sized cube were consistently reinforced by "finding the hole" drilled in the bottom of the middle-sized cube and by E's verbal "good" or "fine."

During training, all Ss were presented with white $1-, 1 \frac{1}{4}-$, and $1 \frac{1}{2}$-in. cubes for 75 trials or until a criterion of five of six correct responses was met. No $S$ failed to reach the criterion within the 75-trial limit.

On reaching the criterion on the training task, Ss were switched, without comment, to the near test (stimuli $=1 \frac{1}{4}, 11 \frac{1}{2}$, and $13 / 4$ in.) or to the far test (stimuli $=13 / 4,2$, and $2 \frac{1}{4}$ in.). Subjects in the control (C) condition continued to work with white cubes, as those in the between-trials (B-T) condition and the within-trials (W-T) condition began working with red, blue, and yellow cubes. Response to the middle-sized cube was reinforced on all 18 test trials.

For $S s$ in the B-T condition, all three cubes used on a trial were the same color. The color of cubes changed from trial to trial so that, in each block of six trials, cubes were red on two trials, blue on two trials, and yellow on two trials. The aim of this manipulation was to make the change from training to test stimuli more noticeable than it was in the $\mathrm{C}$ condition.

Subjects in the W-T condition were exposed to one red, one blue, and one yellow cube on each trial. Each color was paired with each size cube on two trials during each block of six trials (e.g., for all W-T Ss, the middle-sized block was yellow on Trials 1 and 5 , blue on Trials 2 and 4 , and yellow on Trials 3 and 6 on the first block of trials). The aim of this manipulation was to make the change from training to test stimuli more noticeable than in the $C$ condition and to accent the difference among test stimuli.

\section{RESULTS}

Trials to criterion on the training task were analyzed by a 3 (conditions) by 2 (distance) analysis of variance. All of the Fs were $<1$.

The test trials data were analyzed by use of chi square tests and two analyses of variance. The dependent variables were: number of first-trial transposition responses, number of transposition responses during the first six trials, and number of transposition responses over three blocks of six trials each.

The first-trial data shown in Fig. 1 indicated that the frequencies of transposition responses in the B-T and the W-T conditions were identical or nearly

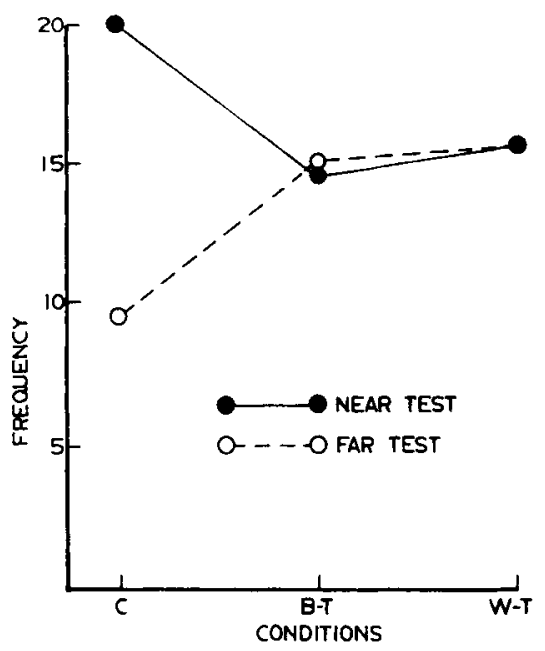

Fig. 1. Transposition responses on the first test trial. 